\title{
Revisiting the past - novel insight into intraspecific molecular diversity and phylogeny of widespread Echinogammarus berilloni
}

\author{
Kamil Hupało* \\ Aquatische Ökosystemforschung, Universität Duisburg-Essen, Universitätsstr. 5, Essen D-45141, Germany
}

Received: 20 July 2020 / Accepted: 18 November 2020

\begin{abstract}
Echinogammarus berilloni (Catta, 1878) is a widespread and euryecious species, inhabiting large parts of Southwestern Europe. Additionally, it is recognized as one of the non-native and invasive species, increasing its range to Central Europe. Even though it is widely used as a model organism in a variety of ecological and ecotoxicological studies, its intraspecific molecular diversity has been overlooked. In this study, the publicly available genetic data reveal high level of intraspecific diversity, supporting presence of up to four phylogenetically distinct genetic lineages. Furthermore, the results indicate that the taxon is not monophyletic. The importance of considering its intraspecific diversity is discussed, highlighting the potential implications for future studies as well as the need for further detailed taxonomic studies.
\end{abstract}

Keywords: Gammaridae / freshwater / cryptic diversity / invasive / Amphipoda

\begin{abstract}
Résumé - Réexamen du passé - un nouvel aperçu de la diversité moléculaire intraspécifique et de la phylogénie du très répandu Echinogammarus berilloni. Echinogammarus berilloni (Catta, 1878) est une espèce très répandue et eurytope qui est présente dans une grande partie de l'Europe du Sud-Ouest. De plus, elle est reconnue comme l'une des espèces non indigènes et envahissantes, qui augmente son aire de répartition en Europe centrale. Bien qu'il soit largement utilisé comme organisme modèle dans diverses études écologiques et écotoxicologiques, sa diversité moléculaire intraspécifique a été négligée. Dans cette étude, les données génétiques accessibles au public révèlent un niveau élevé de diversité intraspécifique, ce qui confirme la présence de jusqu'à quatre lignées génétiques phylogénétiquement distinctes. En outre, les résultats indiquent que le taxon n'est pas monophylétique. L'importance de prendre en compte sa diversité intraspécifique est discutée, en soulignant les implications potentielles pour les études futures ainsi que la nécessité d'études taxonomiques détaillées supplémentaires.
\end{abstract}

Mots-clés : Gammaridae / eau douce / diversité cryptique / envahissant / Amphipoda

\section{Introduction}

Due to the rising anthropogenic activities and ongoing climate change, more and more ecosystems are affected by the migration of non-indigenous species and their impact on native biota (e.g. Rahel and Olden, 2008; Hulme, 2009). The development of trade waterways and man-made canals facilitated spread of numerous freshwater taxa through newly connected river systems. It is particularly pronounced in Europe, where aquatic species have migrated exponentially from the beginning of the twentieth century (Galil et al., 2008). Being a keystone aquatic species, alien amphipods, mostly originating from the PontoCaspian region, have arguably one of strongest impact on the native European freshwater fauna (Grabowski et al., 2007;

\footnotetext{
*Corresponding author: kamilhupalo@gmail.com
}

Hänfling et al., 2011). One of the first recognized gammarid invaders was Echinogammarus berilloni (Catta, 1878), and it is believed to originate from southwestern Europe (Catta, 1878). In the years following, the species was reported from northeast Spain, southwest France and then subsequently from Belgium, Netherlands up to north-western Germany (Pinkster, 1973). Even though E. berilloni shows significant ecological trait advantages over native gammarids (Piscart et al., 2011b; Schmidt-Drewello et al., 2016), these advantages are not as pronounced as in other amphipod invaders present in European fresh waters (Kley and Maier, 2006).

Thanks to the use of extensive molecular data, more is known about both the dispersal history and intraspecific diversity of gammarid invaders (Rewicz et al., 2015) as well as overlooked cryptic diversity of widely dispersed native species (Mamos et al., 2014). On the other hand, little is known about the invasion history and intraspecific diversity of E. berilloni. 
Even though it is used as a model organism in numerous studies focusing on ecotoxicology (Boets et al., 2012), physiology (Shatilina et al., 2011) or ecology (Médoc et al., 2015), the available molecular data for the species, given its vast distribution, is surprisingly scarce and analysed only in broader phylogenetic and ecological context (Hou et al., 2014; Grabner et al., 2015; Sket and Hou, 2018). Thus, the goal of this study was to analyse the available molecular data to provide first insight on intraspecific diversity of E. berilloni, and to verify if the species shows signs of cryptic diversity.

\section{Material and methods}

Analysed molecular material was composed of 20 DNA sequences deriving from individuals identified as Echinogammarus berilloni, all publicly available and mined from GenBank, belonging to mitochondrial cytochrome oxidase I (COI) gene (9 sequences) and to nuclear markers: 28S rRNA (6 sequences) and $18 \mathrm{~S}$ rRNA ( 5 sequences) (all listed in Tab. S1 and aggregated together in Barcode of Life Database [BOLD; Ratnasingham and Hebert, 2007] http://dx.doi.org/10.5883/DS-EBER). The obtained sequences originate from broad distributional range, from individuals collected from Spain, Germany and France, including species' locus typicus (Fig. 1, Tab. S1). Obtained sequences were assembled and aligned in Geneious software (Biomatters, 2013). The alignment was performed using MAFFT plugin (Katoh and Standley, 2013). The haplotype networks for each molecular marker used in this study were done using Minimum Spanning Networks in POPART software (Leigh and Bryant, 2015). The K2p pairwise genetic distances for all studied molecular markers were calculated in MEGA7 software (Kumar et al., 2016). Molecular Operational Taxonomic Units (MOTUs) were delimited using multilocus species delimitation algorithm STACEY as a primary delimitation method, including both mitochondrial and nuclear information available (Jones, 2017). The results of the STACEY delimitation were cross-validated with COI-based delimitation methods like ABGD (Puillandre et al., 2012), Generalized Mixed Yule Coalescent, single and multiple models (GMYC; Pons et al., 2006) and a Bayesian implementation of the Poisson tree processes (bPTP; Zhang et al., 2013). All technical details of MOTU delimitation analyses performed including the input trees, parameters used and detailed cross-validation of obtained delimitation results are described in Supplementary File 1.

The final dataset used for reconstruction of time-calibrated phylogeny consisted of a single representative individual per each delimited MOTU, according to STACEY delimitation algorithm complemented with multiple reference sequences belonging to congeners and related genera, as well as individuals which were used for calibration of the molecular clock (all of them listed in Supplementary File 2). No significant saturation was detected for any molecular marker and the dataset was divided into five partitions: $28 \mathrm{~S}, 18 \mathrm{~S}$ as single partitions with three codon positions of COI. The timecalibrated phylogeny was reconstructed in BEAST 2.4.7 package (Bouckaert et al., 2014), performing three MCMC chains of 100 million iterations, sampled every 2000 iterations, using the best-fit substitution models determined by bModel test (Bouckaert and Drummond, 2017), Yule model as a tree prior and uncorrelated exponential relaxed clock. The molecular clock was calibrated using five primary calibration points known from literature and related to geological events (e.g., emergence of Lake Ohrid, separation of Black and Caspian seas or Miocene subsidence event). The divergence times were cross-validated with the ones obtained using the amphipod-specific substitution rates deriving from fossil evidence (Copilas-Ciocianu et al., 2019). Afterwards, the parameters of all runs were examined in Tracer 1.7.1 and reached the ESS values above 200. The runs were combined and resampled with the maximum clade credibility chronograms being annotated and visualized. All details regarding the reference material, dataset handling, calibration schemes and analytical parameters used are described in Supplementary File 2 (software, approaches and reference material used: Sherbakov, 1999; Cristescu et al., 2003; Xia et al., 2003; Cristescu and Hebert, 2005; Macdonald Iii et al., 2005; Drummond et al., 2006; Hou et al., 2011, 2014; Mats et al., 2011; Lanfear et al., 2012; Nahavandi et al., 2013; Wysocka et al., 2013; Bouckaert et al., 2014; Wysocka et al., 2014; Copilas-Ciocianu and Petrusek, 2015; Bouckaert and Drummond, 2017; Sket and Hou, 2018; Xia, 2018; Copilas-Ciocianu et al., 2019).

\section{Results}

The STACEY delimitation algorithm supported presence of four distinct lineages within studied E. berilloni dataset, one being composed solely with an individual originating from the locus typicus, one being composed of individuals from France and Germany and two being present in the material deriving from Spain (Fig. 1). Other delimitation methods generally supported the distinctiveness of the delimited lineages, with the exception of the multiple threshold GMYC, which delineated only two lineages (Supplementary File 1).

The analysis of haplotype networks and $\mathrm{K} 2 \mathrm{p}$ pairwise distances revealed no shared haplotypes between the studied individuals, both on mitochondrial and nuclear level (Fig. 1, Supplementary File 2). The COI genetic distances between the delimited MOTUs largely exceeded the maximal intraspecific threshold of 0.0309 proposed before (Costa et al., 2007). The genetic distances for nuclear markers were generally lower than the one observed in the mitochondrial data, given the conservativeness of the studied nuclear markers.

The time-calibrated phylogeny revealed presence of two monophyletic clades, one comprising of MOTUs Eb1 and Eb2 and second with Eb3 and Eb4, respectively. Although, the phylogenetic reconstruction supports the monophyly of socalled Echinogammarus berilloni group, it does not support the monophyly of all four E. berilloni MOTUs grouped within and indicate that the diversification of their common ancestor probably took place in Miocene (95\% HPD: 26.8-5.9 Ma). The divergence events within those two groups seemed to take place more recently, likely in Plio-Pleistocene (95\% HPD: 11.7-0.4 Ma for Eb1, Eb2; 8.1-1.0 Ma for Eb3, Eb4). The divergence times obtained using both primary calibration points and the substitution rates delivered congruent results, described in detail in Supplementary File 2.

\section{Discussion}

Despite the low number of analysed individuals, the obtained results support a high level of overlooked intraspecific diversity within E. berilloni. Even though the 
K. Hupało: Knowl. Manag. Aquat. Ecosyst. 2020, 421, 46

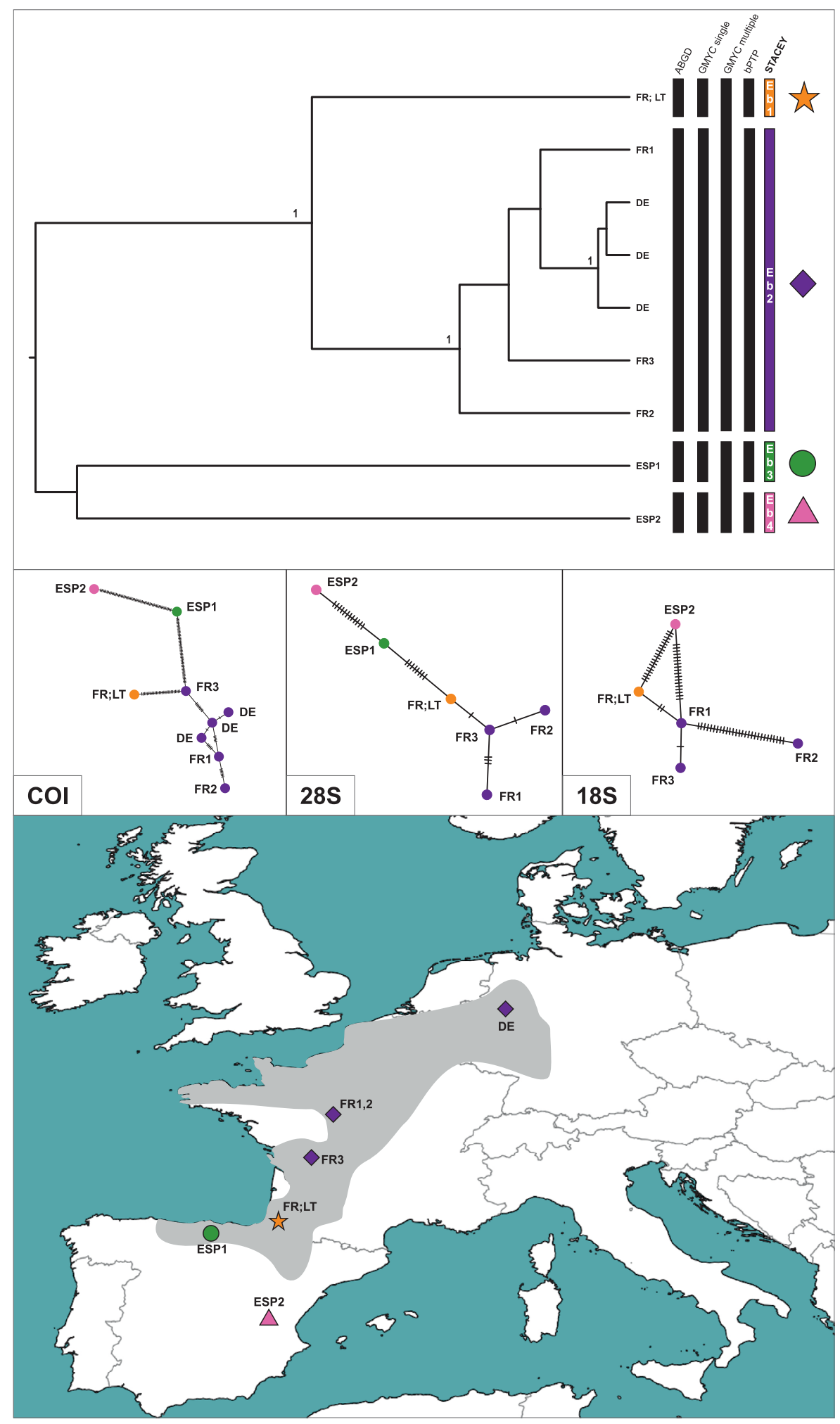

Fig. 1. Maximum clade credibility tree of the Echinogammarus berilloni mined from NCBI GenBank, obtained from STACEY multilocus species delimitation analysis. Phylogeny was inferred from sequences of the mitochondrial COI and nuclear: 28S rRNA and 18S rRNA markers. The numbers by respective nodes indicate Bayesian posterior probability values $=1$. The rows of respective bars represent the delimitation of molecular operational taxonomic units (MOTU) by various methods of species delimitation. The coloured symbols on the map represent STACEY-delimited MOTUs. The minimum spanning haplotype networks were generated using POPART software. The grey area on the map reflects known distribution range of E. berilloni. The star symbol corresponds to the individual collected in species' locus typicus (marked with 'LT' on phylogenetic tree). The map was generated using QGIS 2.18.3 (https://www.qgis.org/en/site/). 


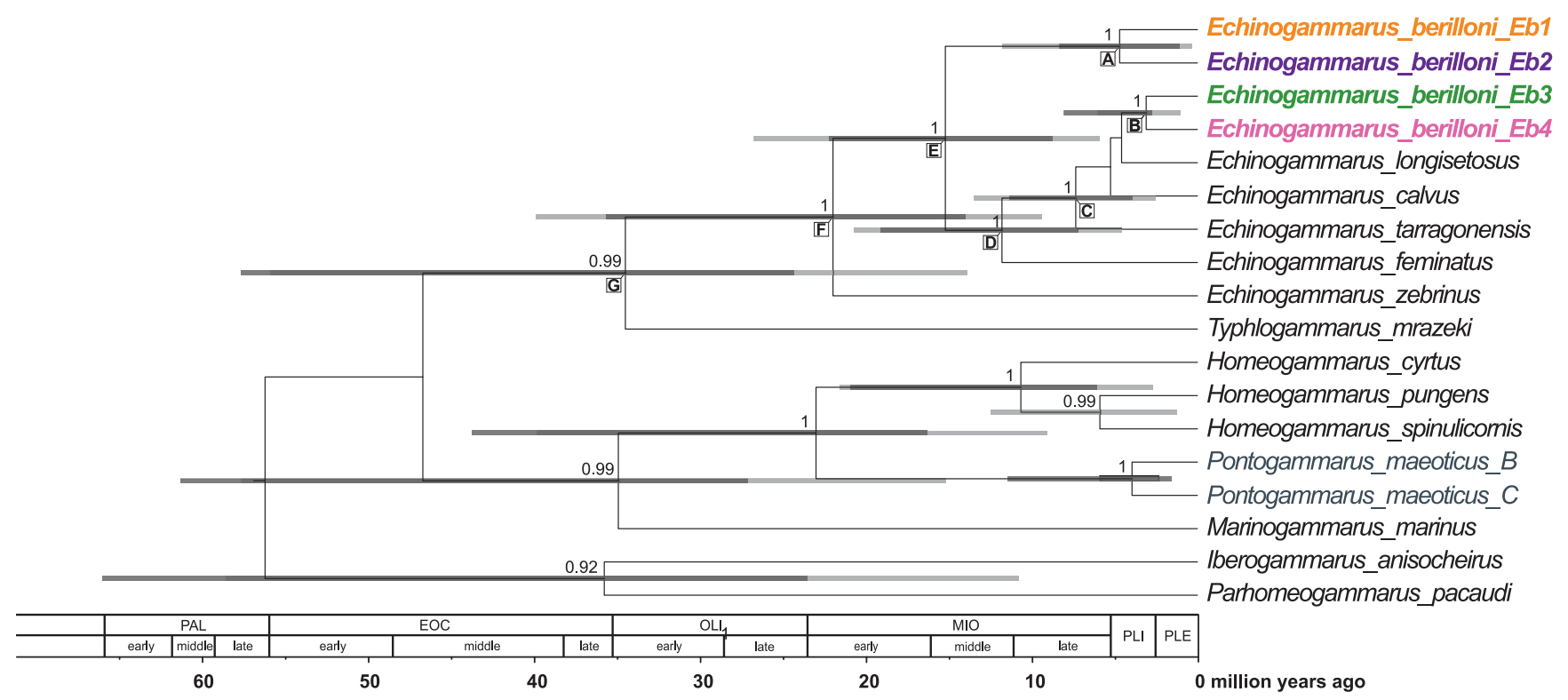

Fig. 2. Maximum clade credibility, time-calibrated Bayesian reconstruction of phylogeny of Echinogammarus berilloni MOTUs along with the reference material. Phylogeny was inferred from sequences of the mitochondrial COI and nuclear: 28S rRNA and 18S rRNA markers. The numbers by respective nodes indicate Bayesian posterior probability values $>0.9$. The colours of respective MOTUs of $E$. berilloni correspond to those presented in Figure 1. The grey species' names as well as the highlighted nodes correspond to the sequences used for calibrating the molecular clock. Grey node bars represent 95\% HPD values: light grey represent the 95\% HPD values obtained using primary calibration scheme whereas dark grey bars indicate the $95 \%$ HPD values obtained using substitution rates from the literature. Further information about the divergence times of selected nodes, marked with letters, are available in Supplementary File 2.

delimitation methods used in this study are not conclusive, there are all supporting presence of more than one distinct species in the analysed dataset. The majority of the implemented delimitation methods supported distinctiveness of the individual of $E$. berilloni deriving from the population inhabiting locus typicus (Fig. 1; Supplementary File 1). Even though the differences in genetic distances between the individual from this population and others in France based on mitochondrial DNA are profound, they are much lower on the nuclear level (Fig. 1; Tab. S2). This might suggest the possibility of recent connectivity between those populations, however, no shared nuclear haplotypes were found, which for now, do not support the ongoing gene flow. However, although the number of individuals analysed in this study is scarce, the complete gene sorting from mitochondrial and nuclear DNA might indicate the validity of observed diversity (Zink and Barrowclough, 2008). Thus, it might serve as a starting point for further thorough studies of the various populations of this species, leading to a possibility of its redescription.

Moreover, the molecular differences can be already associated with some morphological differences, remarked by the authors for both individuals from Spain, which were 'less setose' than the other studied individuals (Sket and Hou, 2018). Although there is no available information about any further morphological features in other studied populations, the morphological variability within E. berilloni was known and discussed for years. In fact, morphological incongruence have led to the description of several new species, which were all formerly assigned to E. berilloni (Catta, 1878; Pinkster, 1973). Even after the species redescription, E. berilloni was still characterized by significant level of both seasonal and regional variability in the characters like shape of the eyes, shape of the epimeral plates, the armature of the telson lobes and the length and number of setae and spines present (Pinkster, 1973, 1993).

Interestingly, the results of the time-calibrated phylogeny support lack of monophyly between E. berilloni MOTUs present in France and Germany and the ones reported from Iberian Peninsula (Fig. 2). Since all other known members of E. berilloni group are presumably originating from Iberian Peninsula and presently distributed only there (Pinkster, 1973), one could argue that all E. berilloni lineages might have originated in Iberian Peninsula as well and then disperse northwards. However, the timing of divergence suggests the split of those two groups already during Miocene, indicating that the lineages present today north of Pyrenees might have evolved and diversified already outside of Iberian Peninsula. Still, it is hard to confidently resolve how the gammarid ancestor species might have crossed the Pyrenees. Theoretically, it could have been possible to migrate outside of Iberian Peninsula using land connection, since Pyrenees at that time did not isolate the entire Peninsula (e.g. Popov et al., 2004). Although the faunal exchange in Miocene between Iberian Peninsula and Central Europe has been discussed and confirmed for mammals (Casanovas-Vilar et al., 2010), there is arguably no evidence on similar patterns observed in freshwater taxa nor the connectivity between the river networks at that time. Thus, one cannot exclude the possibility of a passive dispersal by means of ectozoochory already discussed for Iberian gammarid amphipods ((Rachalewski et al., 2013). Another possible scenario would be an active dispersal via marine waters, already proven plausible for Echinogammarus berilloni as it can tolerate substantial levels of elevated salinity (Piscart et al., 2011a). The results suggest 
that the diversification within two $E$. berilloni groups probably took place during late Miocene or in Pliocene (Fig. 2). Given the current distribution of the members of supported lineages, one might argue that the diversification events might have been triggered by recurrent changes in water networks on Iberian Peninsula due to Messinian Salinity Crysis and/or due to erosion of Iberian Maseta and Massif Central, provided that some of the lineages might have crossed the Pyrenees already in Miocene (Popov et al., 2004).

The results presented here provide a valuable taxonomic insight into the studied species as well as the entire Echinogammarus berilloni group. The multilocus species delimitation and the reconstructed phylogeny both support presence of more than one taxonomical entity within E. berilloni species (Figs. 1 and 2). The results of this study also significantly change the views on species' distribution. Since the Eb1 lineage is so far known only from the species' locus typicus with two Iberian lineages, Eb3 and Eb4, being known from limited areas of Spain, the Eb2 lineage is arguably the only one being distributed on a wider area in Central Europe (Fig. 1). Even though the sample material analysed here is limited, it already indicates the probable shift in the actual distribution area compared to the one presented before (Pinkster, 1993), the phenomenon already observed in other widely distributed amphipod species in Europe (e.g. Mamos et al., 2016; Grabowski et al., 2017; Wattier et al., 2020). Obtained results also support the need for further taxonomical studies on Echinogammarus berilloni involving the comparative morphological analyses, taking into account reported species' variability of multiple diagnostic characters (Pinkster, 1973).

The results presented here also add yet another piece of evidence questioning the invasiveness of Echinogammarus berilloni in Central Europe. Despite few studies indicating its advantage over native gammarid species with confirmed cases of their displacement, E. berilloni does not seem to exhibit all the traits assigned to an invasive gammarid (Grabowski et al., 2007; Schmidt-Drewello et al., 2016). The results presented here indicate that only Eb2 lineage has managed to disperse east of France and given what is known for the moment about its distribution, it rejects the concept of a recent dispersal directly from Iberian Peninsula. The findings of several experimental studies conducted across distribution area of $\mathrm{Eb} 2$ lineage focusing on life history traits of E. berilloni indicated high reproductive potential, increased salinity tolerance and advantage in fish predation, however there was no direct evidence supporting direct displacement of other gammarid species (e.g. Piscart et al., 2011a; Médoc et al., 2015; SchmidtDrewello et al., 2016), also in some cases treating E. berilloni as native to the region (e.g. Piscart et al., 2007; Piscart et al., 2011a,b). Although sightings of this species reported throughout 20th century from Germany, Belgium, Netherlands and Luxembourg suggest its recent expansion, the lack of shared mitochondrial haplotypes between populations from France and Germany presented here questions that assumption. In case of a recent expansion, one would expect diversity patterns observed for example in Dikerogammarus villosus, a well-known recent gammarid invader (Rewicz et al., 2015). The haplotype diversity presented here suggests rather that, although the expansion might have been facilitated by establishment of artificial waterways connecting Loire and Rhine in the 19th Century, the distribution and the gene flow might have been seized already for some time. However, to resolve that matter, more molecular evidence is needed, especially from nuclear markers from the population present easternmost, lacking in currently analysed dataset. That is why the invasiveness of $E$. berilloni is put in doubt, but one cannot exclude the possibility that only single lineage (in this case Eb2) might present invasive potential. However, since genetic diversity was not tested in those experimental studies, it is hard to confidently resolve that matter.

In conclusion, the results of this study present an analysis of molecular evidence on the long overlooked intraspecific diversity in E. berilloni. This research serves as a solid starting point for future studies on species' taxonomy and ecology. It also highlights the urgent need for further comprehensive molecular and detailed morphological studies, particularly in the framework of integrative taxonomy, combining both morphological and molecular characters. The findings of this study also have implications for future experimental research on this species. It is known that the cryptic species belonging to the same species complex could respond differently to various stressors (Fišer et al., 2018 and references therein). Given that $E$. berilloni is being studied extensively across its distributional range (Médoc et al., 2015; Schmidt-Drewello et al., 2016; Solagaistua et al., 2019), the implementation of molecular identification of the studied individuals seems necessary to fully understand the invasiveness of the species and to provide a better comparability and reliability of obtained results.

\section{Supplementary Material}

Supplementary File 1. MOTU delimitation (Tables S1.1 to S1.4)

Supplementary File 2. Time-calibrated phylogeny (Tables S2.1 to S2.6 and Figure S2.1)

Table S1. Material of Echinogammarus berilloni used in this study

Table S2. Genetic pairwise K2p distances calculated for individuals amplified for both mitochondrial and nuclear molecular markers

The Supplementary Material is available at https://www.kmaejournal.org/10.1051/kmae/2020038/olm.

Acknowledgments. The author would like to thank Dr. Kristy Deiner, who provided valuable comments and kindly agreed to perform language corrections. The author was supported by the Polish National Science Center (projects no. 2018/28/T/NZ8/ 00022) and the German Research Foundation (DFG) (project LE 2323/9-1).

\section{References}

Boets P, Lock K, Goethals PL, Janssen CR, De Schamphelaere KA. 2012. A comparison of the short-term toxicity of cadmium to indigenous and alien gammarid species. Ecotoxicology 21: $1135-1144$. 
Bouckaert R, Heled J, Kühnert D, Vaughan T, Wu C-H, Xie D, Suchard MA, Rambaut A, Drummond AJ. 2014. BEAST 2: a software platform for Bayesian evolutionary analysis. PLoS Comput. Biol. 10.

Bouckaert RR, Drummond AJ. 2017. bModelTest: Bayesian phylogenetic site model averaging and model comparison. BMC Evol. Biol. 17: 42.

Casanovas-Vilar I, García-Paredes I, Alba DM, van den Hoek Ostende LW, Moyà-Solà S. 2010. The European Far West: Miocene mammal isolation, diversity and turnover in the Iberian Peninsula. J. Biogeogr. 37: 1079-1093.

Catta J-D. 1878. Note sur le Gammarus berilloni (n. sp.). Bull. Soc. Borda. 1: 69-73.

Copilas-Ciocianu D, Petrusek A. 2015. The southwestern Carpathians as an ancient centre of diversity of freshwater gammarid amphipods: insights from the Gammarus fossarum species complex. Mol. Ecol. 24: 3980-3992.

Copilas-Ciocianu D, Sidorov D, Gontcharov A. 2019. Adrift across tectonic plates: molecular phylogenetics supports the ancient Laurasian origin of old limnic crangonyctid amphipods. Org. Divers. Evol. 19: 191-207.

Costa FO, DeWaard JR, Boutillier J, Ratnasingham S, Dooh RT, Hajibabaei M, Hebert PD. 2007. Biological identifications through DNA barcodes: the case of the Crustacea. Can. J. Fish. Aquat. Sci. 64: 272-295.

Cristescu ME, Hebert PD, Onciu TM. 2003. Phylogeography of Ponto-Caspian crustaceans: a benthic-planktonic comparison. Mol. Ecol. 12: 985-996.

Cristescu ME, Hebert PD. 2005. The "Crustacean Seas" an evolutionary perspective on the Ponto Caspian peracarids. Can. J. Fish. Aquat. Sci. 505-517.

Drummond AJ, Ho SY, Phillips MJ, Rambaut A. 2006. Relaxed phylogenetics and dating with confidence. PLoS Biol. 4.

Fišer C, Robinson CT, Malard F. 2018. Cryptic species as a window into the paradigm shift of the species concept. Mol. Ecol. 27: 613-635.

Galil BS, Nehring S, Panov V. 2008. Waterways as invasion highways-impact of climate change and globalization. Biol. Invasions 193: 59-74.

Grabner DS, Weigand AM, Leese F, Winking C, Hering D, Tollrian R, Sures B. 2015. Invaders, natives and their enemies: distribution patterns of amphipods and their microsporidian parasites in the Ruhr Metropolis, Germany. Parasit. Vectors 8: 419.

Grabowski M, Bacela K, Konopacka A. 2007. How to be an invasive gammarid (Amphipoda: Gammaroidea)-comparison of life history traits. Hydrobiologia 590: 75-84.

Grabowski M, Mamos T, Bącela-Spychalska K, Rewicz T, Wattier RA. 2017. Neogene paleogeography provides context for understanding the origin and spatial distribution of cryptic diversity in a widespread Balkan freshwater amphipod. PeerJ 5: e3016.

Hänfling B, Edwards F, Gherardi F. 2011. Invasive alien Crustacea: dispersal, establishment, impact and control. BioControl 56: 573-595.

Hou Z, Sket B, Fišer C, Li S. 2011. Eocene habitat shift from saline to freshwater promoted Tethyan amphipod diversification. Proc. Natl. Acad. Sci. 108: 14533-14538.

Hou Z, Sket B, Li S. 2014. Phylogenetic analyses of Gammaridae crustacean reveal different diversification patterns among sister lineages in the Tethyan region. Cladistics 30: 352-365.

Hulme PE. 2009. Trade, transport and trouble: managing invasive species pathways in an era of globalization. J. Appl. Ecol. 46: $10-18$.
Jones G. 2017. Algorithmic improvements to species delimitation and phylogeny estimation under the multispecies coalescent. J. Math. Biol. 74: 447-467.

Katoh K, Standley DM. 2013. MAFFT multiple sequence alignment software version 7: improvements in performance and usability. Mol. Biol. Evol. 30: 772-780.

Kley A, Maier G. 2006. Reproductive characteristics of invasive gammarids in the Rhine-Main-Danube catchment, South Germany. Limnologica 36: 79-90.

Kumar S, Stecher G, Tamura K. 2016. MEGA7: molecular evolutionary genetics analysis version 7.0 for bigger datasets. Mol. Biol. Evol. 33: 1870-1874.

Lanfear R, Calcott B, Ho SY, Guindon S. 2012. PartitionFinder: combined selection of partitioning schemes and substitution models for phylogenetic analyses. Mol. Biol. Evol. 29: 1695-1701.

Leigh JW, Bryant D. 2015. POPART: full-feature software for haplotype network construction. Methods Ecol. Evol. 6: $1110-1116$.

Macdonald Iii KS, Yampolsky L, Duffy JE. 2005. Molecular and morphological evolution of the amphipod radiation of Lake Baikal. Mol. Phylogenet. Evol. 35: 323-343.

Mamos T, Wattier R, Majda A, Sket B, Grabowski M. 2014. Morphological vs. molecular delineation of taxa across montane regions in Europe: the case study of Gammarus balcanicus Schäferna, (Crustacea: A mphipoda). J. Zool. Syst. Evol. Res. 52: 237-248.

Mamos T, Wattier R, Burzyński A, Grabowski M. 2016. The legacy of a vanished sea: a high level of diversification within a European freshwater amphipod species complex driven by $15 \mathrm{My}$ of Paratethys regression. Mol. Ecol. 25: 795-810.

Mats V, Shcherbakov DY, Efimova I. 2011. Late CretaceousCenozoic history of the Lake Baikal depression and formation of its unique biodiversity. Stratigr. Geol. Correl. 19: 404.

Médoc V, Albert H, Spataro T. 2015. Functional response comparisons among freshwater amphipods: ratio-dependence and higher predation for Gammarus pulex compared to the nonnatives Dikerogammarus villosus and Echinogammarus berilloni. Biol. Invasions 17: 3625-3637.

Nahavandi N, Ketmaier V, Plath M, Tiedemann R. 2013. Diversification of Ponto-Caspian aquatic fauna: Morphology and molecules retrieve congruent evolutionary relationships in Pontogammarus maeoticus (Amphipoda: Pontogammaridae). Mol. Phylogenet. Evol. 69: 1063-1076.

Pinkster S. 1973. The Echinogammarus berilloni-group, a number of predominantly Iberian amphipod species (Crustacea). Bijdr. Dierkd. 43: 1-38.

Pinkster S. 1993. A revision of the genus Echinogammarus Stebbing, 1899, with some notes on related genera (Crustacea, Amphipoda). Memorie del Museo Civ. Stor. nat.(IIa ser.).

Piscart C, Manach A, Copp GH, Marmonier P. 2007. Distribution and microhabitats of native and non-native gammarids (Amphipoda, Crustacea) in Brittany, with particular reference to the endangered endemic sub-species Gammarus duebeni celticus. J. Biogeogr. 34: $524-533$.

Piscart C, Kefford BJ, Beisel J-N. 2011a. Are salinity tolerances of non-native macroinvertebrates in France an indicator of potential for their translocation in a new area? Limnologica 41: 107-112.

Piscart C, Roussel JM, Dick JT, Grosbois G, Marmonier P. $2011 \mathrm{~b}$. Effects of coexistence on habitat use and trophic ecology of interacting native and invasive amphipods. Freshw. Biol. 56: 325-334.

Pons J, Barraclough TG, Gomez-Zurita J, Cardoso A, Duran DP, Hazell S, Kamoun S, Sumlin WD, Vogler AP. 2006. Sequence- 
based species delimitation for the DNA taxonomy of undescribed insects. Syst. Biol. 55: 595-609.

Popov SV, Rögl F, Rozanov AY, Steininger FF, Shcherba IG, Kovac M. 2004. Lithological-paleogeographic maps of Paratethys-10 maps late Eocene to pliocene. Courier Forschungsinstitut Senckenberg 250: 1-46.

Puillandre N, Lambert A, Brouillet S, Achaz G. 2012. ABGD, Automatic Barcode Gap Discovery for primary species delimitation. Mol. Ecol. 21: 1864-1877.

Rachalewski M, Banha F, Grabowski M, Anastácio PM. 2013. Ectozoochory as a possible vector enhancing the spread of an alien amphipod Crangonyx pseudogracilis. Hydrobiologia 717: 109-117.

Rahel FJ, Olden JD. 2008. Assessing the effects of climate change on aquatic invasive species. Conservation biology 22: 521-533.

Ratnasingham S, Hebert PD. 2007. BOLD: The Barcode of Life Data System (http://www.barcodinglife.org). Mol. Ecol. notes 7: 355-364.

Rewicz T, Wattier R, Grabowski M, Rigaud T, Bącela-Spychalska K. 2015. Out of the Black Sea: phylogeography of the invasive killer shrimp Dikerogammarus villosus across Europe. PLoS one 10.

Schmidt-Drewello A, Riss HW, Scharsack JP, Meyer EI. 2016. Relative benefit of the invasive Echinogammarus berilloni (Catta, 1878) over native gammarids under fish predation (Gasterosteus aculeatus Linnaeus, 1758). Aquat. Ecol. 50: 75-85.

Shatilina ZM, Riss HW, Protopopova MV, Trippe M, Meyer EI, Pavlichenko VV, Bedulina DS, Axenov-Gribanov DV, Timofeyev MA. 2011. The role of the heat shock proteins (HSP70 and sHSP) in the thermotolerance of freshwater amphipods from contrasting habitats. J. Therm. Biol. 36: 142-149.

Sherbakov DY. 1999. On the phylogeny of Lake Baikal amphipods in the light of mitochondrial and nuclear DNA sequence data. Crustaceana 72: 911-919.
Sket B, Hou Z. 2018. Family Gammaridae (Crustacea: Amphipoda), mainly its Echinogammarus clade in SW Europe. Further elucidation of its phylogeny and taxonomy. Acta Biol. Slov. 61: 93-102.

Solagaistua L, Elosegi A, Larranaga A. 2019. Consumption and performance responses of the amphipod Echinogammarus berilloni change during laboratory incubation. Ann. Limnol. Int. J. Limnol. 25 .

Wattier R, Mamos T, Copilas-Ciocianu D, Jelić M, Ollivier A, Chaumot A, Danger M, Felten V, Piscart C, Žganec K. 2020. Continental-scale patterns of hyper-cryptic diversity within the freshwater model taxon Gammarus fossarum (Crustacea, Amphipoda). Sci. Rep. 10: 1-16.

Wysocka A, Grabowski M, Sworobowicz L, Burzyński A, Kilikowska A, Kostoski G, Sell J. 2013. A tale of time and depth: intralacustrine radiation in endemic Gammarus species flock from the ancient Lake Ohrid. Zool. J. Linn. Soc. 167: 345-359.

Wysocka A, Grabowski M, Sworobowicz L, Mamos T, Burzyński A, Sell J. 2014. Origin of the Lake Ohrid gammarid species flock: ancient local phylogenetic lineage diversification. J. Biogeogr. 41: $1758-1768$.

Xia X. 2018. DAMBE7: new and improved tools for data analysis in molecular biology and evolution. Mol. Biol. Evol. 35: $1550-1552$

Xia X, Xie Z, Salemi M, Chen L, Wang Y. 2003. An index of substitution saturation and its application. Mol. Phylogenet. Evol. 26: $1-7$.

Zhang J, Kapli P, Pavlidis P, Stamatakis A. 2013. A general species delimitation method with applications to phylogenetic placements. Bioinformatics 29: 2869-2876.

Zink RM, Barrowclough GF. 2008. Mitochondrial DNA under siege in avian phylogeography. Mol. Ecol. 17: 2107-2121.

Cite this article as: Hupało K. 2020. Revisiting the past - novel insight into intraspecific molecular diversity and phylogeny of widespread Echinogammarus berilloni. Knowl. Manag. Aquat. Ecosyst., 421, 46. 